\title{
Comparison of the Degree of Conversion of Resin Based Endodontic Sealers Using the DSC Technique
}

\author{
Elisabetta Cottia \\ Paola Scungio ${ }^{\mathrm{a}}$ \\ Claudia Dettoria \\ Guido Ennas ${ }^{b}$
}

\begin{abstract}
Objectives: The aim of this study was to determine the degree of conversion (DC) of three resin based endodontic sealers using the DSC technique.

Methods: The sealers tested were: EndoREZ (ER) (Ultradent, South Jordan, UT); EndoREZ with Accelerator (ER+A) (Ultradent, South Jordan, UT); RealSeal (RS) (SybronEndo, Orange, CA). Two LED units were used to activate the sealers: UltraLume LED 5 (Ultradent, South Jordan, UT, USA); Mini LED Satelec (Satelec Acteon Group, Mérignac Cedex, France). Samples of $4.0 \mathrm{mg}$ were analyzed with a DSC 7 calorimeter (Perkin Elmer Inc., Wellesley, MA, US). Each specimen was irradiated by each lamp four times for 20 seconds at an interval of 2 mins, while the DSC 7 recorded the heat flow developed during the treatment. The degree of conversion and the kinetic curves were calculated from the values of heat developed during each polymerization. The data were statistically analysed with a Kruskal-Wallis one-way ANOVA multiple range and Student-Newman-Keuls (SNK) tests at a $P$ value of .05 .

Results: Statistically significant differences were found in the degree of conversion among the sealers: ER+A showed the highest values with both lamps.

Conclusions: The higher polymerization rate in resin sealers is obtained with the addition of a catalyst. (Eur J Dent 2011;5:131-138)
\end{abstract}

Key words: Resin-Based endodontic sealer; Differential scanning calorimeter; Polymerization; Accelerator; Degree of conversion.

a Department of Conservative Dentistry, University of Cagliari, Cagliari, Italy.

b Professor, Department of Chemical Sciences, University of Cagliari, Cagliari, Italy.

- Corresponding author: Dr. Elisabetta Cotti Department of Conservative Dentistry, University of Cagliari, Via Roma \# 149, 09124, Cagliari, Italy. Phone: 070.662710 Fax: 070.659689

E-mail: cottienddatin.it

\section{INTRODUCTION}

Success in a root canal treatment depends on the complete removal of canal content through mechanical instrumentation, on the accurate disinfection of the endodontic space by chemical agents followed by an adequate three-dimensional obturation. ${ }^{1}$ Gutta-percha in association with different endodontic sealers has been used for long 
time as a traditional root canal obturation system. ${ }^{2}$

Several studies indicated that resin based endodontic sealers exhibit superior sealing ability by adhesion to root-canal walls. ${ }^{3-5}$ In recent years different resin based dual-cure endodontic sealers were introduced to the clinical practice. ${ }^{6}$ EndoREZ (Ultradent, South Jordan, UT) is a urethane dimethacrylate-based dual curing self priming sealer. One of its prime characteristic properties is a high hydrophilicity, allowing the penetration of the material into the dentinal tubules. ${ }^{7}$ This sealer exhibits a good biocompatibility and performs successfully when used as an endodontic sealer together with gutta-percha cones in clinical cases. 8,9 EndoREZ is also compatible when bonding techniques are used, but it polymerizes within 2030 minutes, therefore if the immediate restoration of the tooth is the objective of treatment, the use of a Dimethyl-p-toluidine - based accelerator (UItradent, South Jordan, UT) to be used in conjunction with the sealer has been suggested. ${ }^{10}$

Determination of the degree of conversion (DC) of methacrylate monomers to polymer may be used to evaluate the status of polymerization. The $\mathrm{DC}$ indicates the percentage of monomer-polymer conversion and is both a quantitative and qualitative index of the extent of the polymerization (Ep). ${ }^{11}$

Differential Scanning Calorimetry (DSC) is a thermal analysis technique that provides the variation of Enthalpy in the exothermic reaction of polymerization; thus allowing the examination of the behaviour of the materials tested by means of thermal and physical characterizations. ${ }^{12}$

This exam can be run using small masses of the samples to test ${ }^{11}$ with many advantages: 1) sharper and well defined peaks; 2) less drift from the basis line; 3) a linear proportionality relation between the mass of the specimen and the area under the peak.

DSC is also a convenient method for determining the relative efficacy of the initiator systems, ${ }^{11,13}$ inhibitors ${ }^{11}$ or activators at the end of the polymerization of a given material.

A current problem using some resin based endodontic sealers is their long setting time, ${ }^{14,15}$ probably due to their increased viscosity that may influence the initial reaction rate in the photopolymerization system.

The aim of this study was to determine with the DSC, the DC of three different resin based endodontic sealer systems: EndoREZ (Ultradent, South Jordan, UT, USA); RealSeal (SybronEndo, Orange, CA, USA); and EndoREZ in association with the accelerator (Ultradent, South Jordan, UT, USA), activated with two different LED units: UltraLume LED 5 (Ultradent, South Jordan, UT, USA); Mini LED Satelec (Satelec Acteon Group, Mérignac Cedex, France).

\section{MATERIALS AND METHODS}

In this study three resin based endodontic sealers were used: EndoREZ (ER) (Ultradent, South Jordan, UT, USA); EndoREZ modified with the addition of a Dimethyl-p-toluidine - based accelerator (ER+A) (Ultradent, South Jordan, UT, USA); RealSeal (RS) (SybronEndo, Orange, CA, USA). RealSeal is an endodontic dual curable resin sealer, whose matrix is a mixture of bisphenol-A-glycidyl methacrylate, ethoxylate Bis-GMA, urethane dimethacrylate resin and hydrophilic difunctional matacrylates. ${ }^{16}$

Two different LED light units were used to activate each sealer: UltraLume LED 5 (Ultradent, South Jordan, UT, USA); Mini LED Satelec (Satelec Acteon Group, Mérignac Cedex, France). UltraLume LED 5 lamp produces light with wavelengths between 370 and $500 \mathrm{~nm}$ and a light intensity of $800 \mathrm{~mW} / \mathrm{cm}^{2}$. Mini LED lamp has a wavelengths range between 420 and $480 \mathrm{~nm}$ and it generates a light intensity of $1250 \mathrm{~mW} / \mathrm{cm}^{2}$. In this study we didn't use a radiometer to measure the light intensity of these brand new LED light sources employed, because these parameters were certified by the manufacturers.

Six groups, of three samples each, were therefore created as follows: AU (ER + UltraLume LED 5); BU (RS + UltraLume LED 5); CU (ER + Accelerator + UltraLume LED 5); AM (ER + Mini LED); BM (RS + Mini LED); CM (ER + Accelerator + Mini LED).

A Differential Scanning Calorimeter DSC 7 (Perkin Elmer Inc., Wellesley, MA, USA) was used to evaluate the degree of conversion (DC) of methacrylate monomers to polymer in each specimen.

All materials were prepared and handled under safe softened light before they were cured.

The control lan empty aluminium pan, called “white"), was prepared before DSC measurements, weighed with an analytic balance, placed in the reference sample holder and then irradiated with the same light units at the same conditions 
fixed for the sealers. The exposure of the "white" served to evaluate the heat flow from the lamps.

Specimens were set into previously calibrated DSC standard aluminium pans ( $\varnothing 4.5 \times 2 \mathrm{~mm})$, and weighed using the analytic balance to obtain 4.1$4.2 \mathrm{mg}$ for each material.

To prepare the specimen consisting of ER with the accelerator, a thin layer of accelerator was applied uniformly on the base of each capsule with a micro-brush, then the sealer was placed to fill the capsule until the whole weight of the sample was reached as for all the other specimens.

A custom-made support was built to hold the lamps in a fixed position during polymerization, in order to assure the reproducibility of the DSC measurements, to fully irradiate only the specimen-containing pan, and to keep the distance lamp-specimen constant.

In addition each lamp was inserted inside an isolation cylinder to avoid the dispersion of the beam.

The photo curing units were used by two different polymerization protocols depending on the unit (standard mode for the UltraLume LED 5, and ramping mode for the Mini LED).

Each sample was photopolymerized with 4 irradiations of $20 \mathrm{~s}$ each (the minimum exposure time to obtain an adequate cure) $)^{17}$ at 2 min. interval from each other (this time is necessary to exclude the thermal effect of optical fibres).

The DSC to measure the enthalpy of polymerization was programmed under isothermal conditions at $32^{\circ} \mathrm{C}$, under constant flowing argon environment $(10 \mathrm{ml} / \mathrm{min})$ to avoid oxidation during the polymerization.

After the specimen containing pans were placed in the sample holder, the temperature was immediately increased to $32^{\circ} \mathrm{C}$ la process taking $20 \mathrm{~s}$ ), to start the measurement of the heat flow. The isothermal measurements were therefore always started exactly 2 min after the initial placement of the materials on the aluminium pan so that all the sealers were tested under the same conditions. The heat flow in the DSC was calculated by considering the exact initial weight of the specimens since all the data from each sample were recorded in the software supplied with DSC 7 at the beginning of the experiment.

The heat flow generated during the polymerization reaction was recorded and graphically rep- resented in a kinetic curve by means of the DSC 7 software. The heat of the exothermic reaction obtained from the DSC of each specimen of sealer represented the sum of the exothermic effects caused by the conversion of the monomers and heat flow from the lamp; while the scanning of the "white" (control) represented only the irradiation heat output from the lamp. ${ }^{18}$ The heat of polymerization developed during the test of the "white" was thus subtracted to calculate the heat of polymerization of the specimens.

Enthalpy was calculated from the area under the peak of the isothermal curve, based on the extrapolated baseline at the end of the reaction. The released heat is proportional to the percentage of reacted monomers. ${ }^{19}$ If the cure reaction is the only thermal event, then the reaction rate is proportional to the heat flow. ${ }^{20}$

The kinetic data was elaborated with a KaleidaGraph software system (version 4.0, 2005, Synergy Software, Reading, PA 19606, USA).

A DC value of $100 \%$ was attributed to $E R+A$, which is the sample with maximum enthalpy observed, and the relative degree of conversion of the other sealers was calculated using this as a reference.

The degree of conversion ( $\mathrm{X}^{\text {sample) }}$ was calculated using the following formula:

$$
X^{\text {sample }}=\left(Q \text { s sample } / Q s^{E R+A}\right) \times 100
$$

where Qs sample and Qs ${ }^{E R+A}$ correspond respectively to the values of enthalpy of the sample to test and the sample ER + A used as a reference.

Each group was tested for four repeated irradiation times considering the following factors: material, type of light-curing unit and duration of exposure. Kruskal-Wallis one-way ANOVA multiple range test was performed at a $P$ value of 05 . Student-Newman-Keuls (SNK) test at a P level of .05 was then applied to identify pairwise differences.

\section{RESULTS}

Representative relative values of the degree of conversion as a function of irradiation time measured for the materials tested, irradiated with UltraLume LED 5 and Mini LED are respectively shown in Figure $1 \mathrm{~A}, \mathrm{~B}, \mathrm{C}$. The results are as follows:

a) Samples tested with UltraLume LED 5: group CU showed a degree of conversion signifi- 
cantly higher compared with the other sealers; no statistical differences were found between groups $\mathrm{AU}$ and BU (Figure 1A).

b) Samples tested with Mini LED: statistically significant differences were observed between relative DC of all the materials tested, with the highest values for group $\mathrm{CM}$ and the lowest recorded values for group BM (Figure 1B).

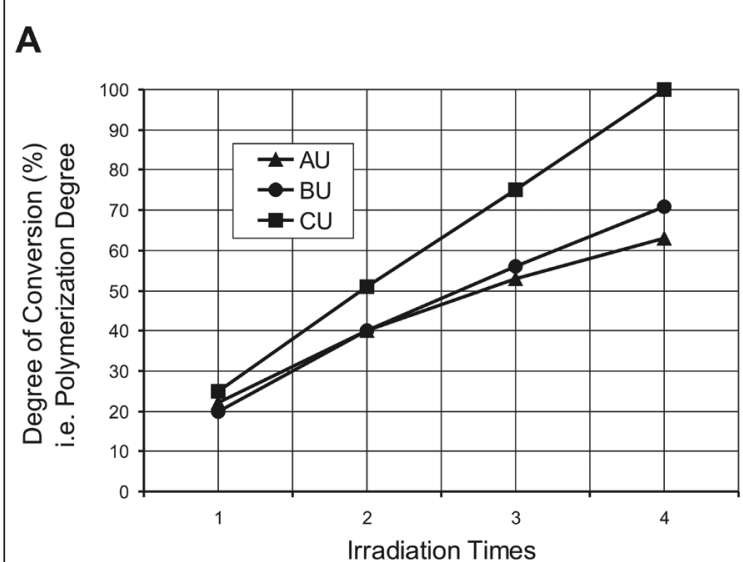

B

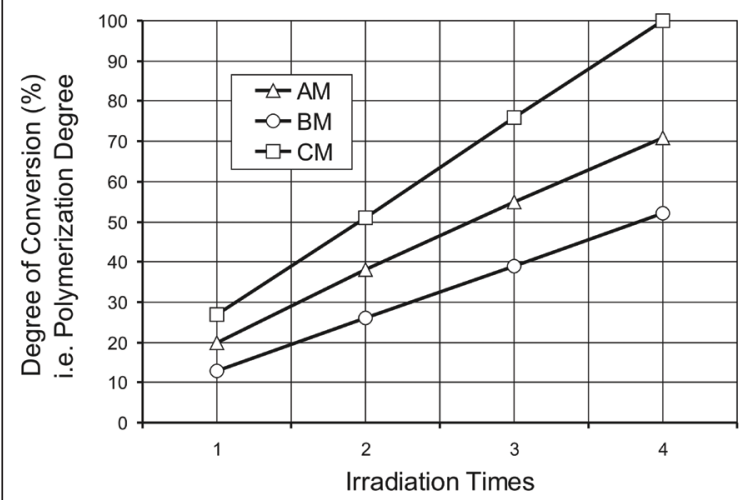

C

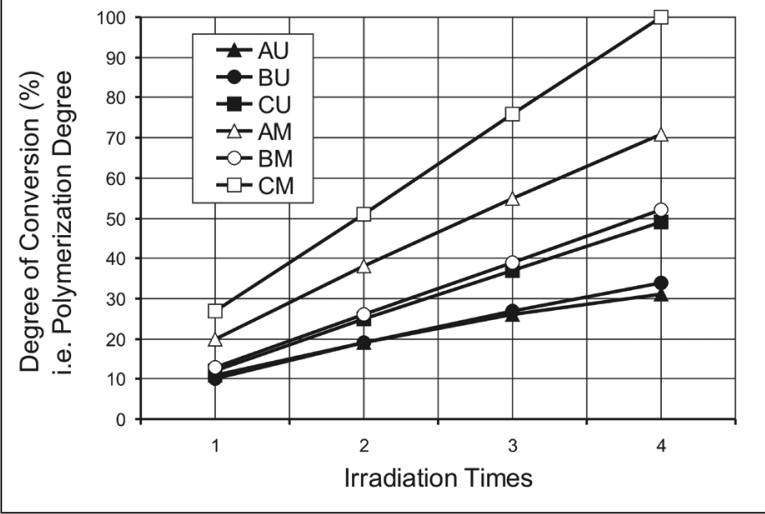

Figure 1. Relative degree of conversion of each specimen as a function of the time obtained with UltraLume LED 5 (A) and Mini LED (B). A comparison among degree of conversion values calculated as a function of irradiation time regardless the curing unit employed (C).
Therefore, the values of enthalpy of the groups $\mathrm{ER}+$ Accelerator (CM and CU) among the specimens polymerized were higher when compared to the other resin materials tested with the same lamp (Figure 2A).

Relative DC values were calculated for each specimen as a function of the irradiation time using as only reference the highest value obtained with UltraLume LED 5 for the specimen ER + A (CU) to which was attributed $100 \%$ of value. Then the relative degree of conversion values were calculated for each specimen using as only reference the highest value obtained with Mini LED for the specimen ER + A (CM) to which was attributed $100 \%$ of value.

Assuming that the heat of reaction corresponds to the extent of the polymerization (Ep) obtained, statistically significant differences in the degree of conversion were observed in the pairwise comparison between the materials tested and the sealers prepared with the addition of the catalyst. According to the data recorded for each material the group CM (ER + Accelerator + Mini LED) showed the highest values of Enthalpy fol-

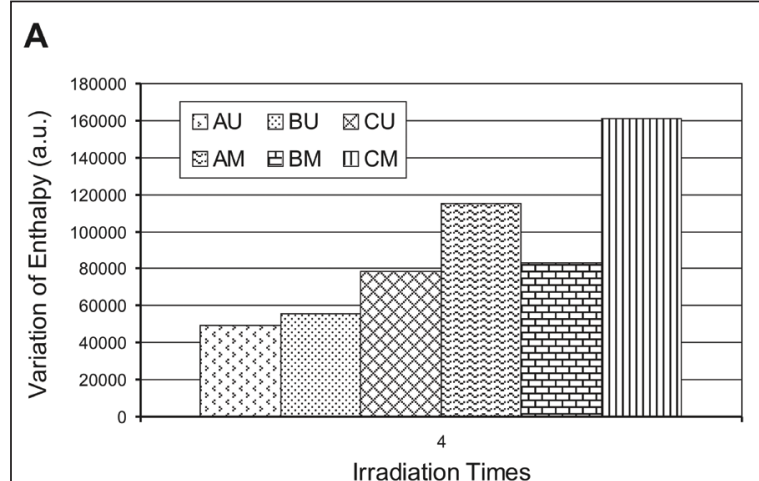

B

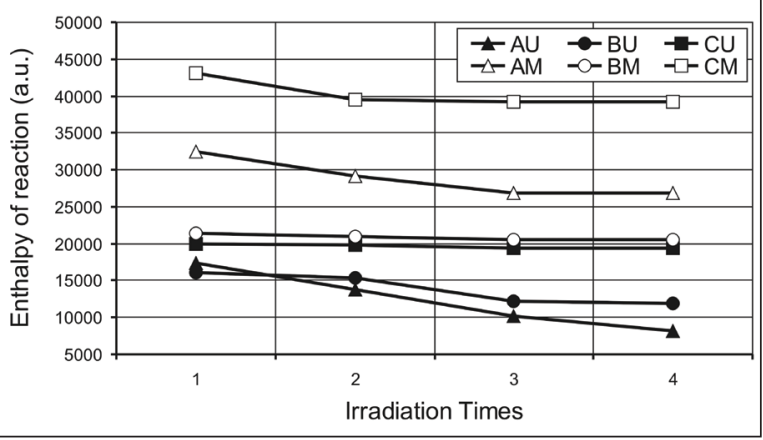

Figure 2. Enthalpy recorded for each sealer tested with the two lamps after four irradiation times (A). Enthalpy measurements after each exposure time of $20 \mathrm{~s}$ (B). 
lowed (in decreasing values of enthalpy) by these groups: AM (ER + Mini LED), BM (RS + Mini LED), CU (ER + Accelerator + UltraLume LED 5), BU (RS + UltraLume LED 5), AU (ER + UltraLume LED 5) (Table 1). The use of the Mini LED produced values of enthalpy of reaction higher than those produced with the UltraLume LED 5 in all the materials tested.

Figure $1 \mathrm{C}$ is representative of relative $\mathrm{DC}$ values calculated for each specimen as a function of irradiation time using as only reference the highest value of enthalpy obtained for the specimen $E R+A+M i n i$ LED. Statistically significant differences were observed among the behaviour of all the specimens with the exception of CU/BM and AU/BU (Table 1).

After the first irradiation all materials tested showed a similar behaviour regarding enthalpy values, which decreased relatively slowly after the successive irradiations and reached a plateau between the third and the fourth irradiation time (Figure 2B).

Among the materials tested with the same lamp, ER+A showed the best values of enthalpy produced after the first irradiation time (Figure 2B) which correspond to a higher conversion than that obtained in the other sealers after $20 \mathrm{~s}$ of irradiation time.

\section{DISCUSSION}

In this study we evaluated the polymerization status of two methacrylate-based resin sealers, both self and photo-curing, that are used for root canal obturation: EndoREZ and RealSeal. ${ }^{21,22}$ We also investigated the behaviour of EndoREZ used with an accelerator.

The ultimate properties of dental resins are directly related both to the chemical composition of the dental monomer system and to the degree of its conversion (DC) to polymer. ${ }^{11}$

In general the DC influences the mechanicalphysical properties and clinical performance of dental resins as it has been underlined by many studies, including compressive strength, flexural strength, hardness and wear. ${ }^{23}$ Two other phenomena connected with the polymerization of the resins are volumetric shrinkage and heating. ${ }^{24}$

The adequacy of the polymerization is influenced by many factors such as the distance of the light source from the resin; the type of resin (shade, translucency and thickness, viscosity); ${ }^{25}$ the temperature; the curing and post-irradiation time; ${ }^{24,26}$ the reactivity of the monomer; the types and amounts of inhibitors, the light; the oxygen permeability ${ }^{27}$ and also the type and amount of photo initiator/co-initiator systems. ${ }^{13,28}$

It has also been shown that dynamic heating of the resin samples increases the rate of polymerization resulting in higher conversion. ${ }^{29,30}$ In our study we tested the materials in isothermal conditions. We used DSC because it is one of the most reliable direct methods for analyzing the degree of conversion of resin blends and the kinetics of the curing reaction. ${ }^{18}$ DSC determines the DC based on the assumption that the heat produced during the reaction (enthalpy) is proportional to the percentage of monomers that have reacted..$^{13,19}$ In addition the enthalpy makes it possible to study how different combinations of photo-initiator and coinitiator systems, or a specific activator, affect the final cure when different light sources are used. ${ }^{13}$ In this study we also determined the conversion level as a function of the irradiation time.

Adequate polymerization of dental resins depends also on the intensity of the light source (power density measured in $\mathrm{mW} / \mathrm{cm}^{2}$ ), correct

Table 1. Enthalpy developed during the polymerization reaction and relative degree of conversion of all the materials tested.

\begin{tabular}{|c|c|c|c|c|c|c|c|c|c|}
\hline \multirow[t]{2}{*}{ Specimens } & \multicolumn{4}{|c|}{$\begin{array}{c}\text { Difference of enthalpy } \\
\text { Irradiation times }\end{array}$} & \multirow{2}{*}{$\begin{array}{l}\text { Total enthalpy } \\
\text { recorded after } 4 \\
\text { irradiation }\end{array}$} & \multicolumn{4}{|c|}{$\begin{array}{l}\text { Relative DC }(\%) \\
\text { Irradiation times }\end{array}$} \\
\hline & 1 & 2 & 3 & 4 & & 1 & 2 & 3 & 4 \\
\hline AU (EndoREZ + UltraLume LED 5) & 17378 & 13788 & 10119 & 8173 & 49458 & 11 & 19 & 26 & 31 \\
\hline BU (RealSeal + UltraLume LED 5) & 16034 & 15378 & 12164 & 11957 & 55533 & 10 & 19 & 27 & 34 \\
\hline CU (EndoREZ + Accelerator + UltraLume LED 5) & 19915 & 19767 & 19414 & 19414 & 78510 & 12 & 25 & 37 & 49 \\
\hline AM (EndoREZ + Mini LED) & 32452 & 29105 & 26851 & 26851 & 115259 & 20 & 38 & 55 & 71 \\
\hline BM (RealSeal + Mini LED) & 21341 & 20903 & 20494 & 20494 & 83232 & 13 & 26 & 39 & 52 \\
\hline CM (EndoREZ + Accelerator + Mini LED) & 43110 & 39559 & 39283 & 39283 & 161235 & 27 & 51 & 76 & 100 \\
\hline
\end{tabular}


wavelength of the light and duration of the exposure. ${ }^{25,26}$ If these parameters are not adequate an incomplete polymerization will occur, together with the exhibition of poor physical properties of the material, higher solubility and consequently an early failure. ${ }^{31}$ In our experiment we used ULtraLume LED 5 and Mini LED. Light Emitting Diodes (LED) units produce light with wavelengths between 450 and $490 \mathrm{~nm}$ with a peak at $460 \mathrm{~nm}$ : this energy range is nearly ideal for activating materials that employ camphorquinone as a photoactivator; therefore LEDs require less power to operate. In addition LED units have an extended life and produce less heat thus respecting pulp and gingival tissue. ${ }^{25,31}$ Dental resins cured with blue LEDs show a larger depth of cure, a higher DC and more stable three-dimensional structures than those cured using halogen lamps. ${ }^{32}$

In this experiment the values of conversion of the EndoREZ + Accelerator system were the highest of all materials tested, both with UltraLume LED 5 and Mini LED lamp. In addition, among the materials tested with the same lamp, EndoREZ + Accelerator showed the highest values of enthalpy after the first irradiation time (20 s). With regard to the groups where EndoREZ + Accelerator was tested, the type of LED light unit employed did not influence the experimental results in terms of polymerization rate when compared to the other resin materials tested.

According to our results relative DC progressively increased in all the specimens tested from the first to the fourth irradiation. Further irradiations after the first light exposure of 20 s contributed to increase the percentage of cured monomers.

EndoREZ with or without Accelerator and RealSeal polymerized with the Mini LED showed a better behaviour than the same sealers polymerized with the UltraLume LED 5: this was probably due to the major intensity of the light source. RealSeal polymerised with the UltraLume LED 5 showed values of conversion from the second to the fourth irradiation that were higher than those obtained from the EndoREZ polymerized with the same lamp, but these differences were not statistically significant. In the group RealSeal + Mini LED at each irradiation time the conversion was significantly lower than that recorded for the group EndoREZ + Mini LED, and the values of enthalpy were almost constant during all the polymerization.
The different behaviour among the materials tested may be due to their different components, such as photo initiators, that allow a different response of the material to the irradiation.

It was not possible to use the same curing mode for the two light units. However in consideration of the exponential increase of the DC as a function of irradiation time, we did not expect significant differences among the two curing modes. In this study we evaluated the differences in DC of the sealers considering the overall characteristics of the LED light units.

During the interval between each irradiation the materials did not exhibit significant exothermic signals, thus indicating that the auto-polymerization was very slow and did not influence this experiment.

The sample preparation consisting of ER with the accelerator was meant to reflect the situation that occurs in the endodontic system where the contact of the activator with ER interests only the interface of the sealer with the gutta-percha cones that are immersed into the accelerator before their placement in the canal previously filled with the EndoREZ.

The higher values of DC exhibited by EndoREZ used in conjunction with the accelerator has a clinical relevance because it decreases the setting time of the material making it possible to complete the restoration of the tooth in one visit. It has also several advantages in terms of increasing the mechanical properties of the material lobturation stability, durability and performance after initial polymerization). It must be considered that a higher polymerization corresponds to a major shrinkage of the resin, which may represent an important cause of microleakage: in a recent study, higher leakage was found when using EndoREZ sealer with Accelerator than EndoREZ alone in delayed post space preparation. ${ }^{10,33}$ On the other hand the placement of many accessory cones, as the surface of the canal allows, reduces the amount of sealer, resulting in less polymerization shrinkage. If the immediate completion of the restoration of a tooth is the objective, then the accelerator should be used.

In the methacrylate-based sealers after light activation residual unreacted monomers continue to react slowly even at body temperature. As these unreacted monomers are responsible for the bio- 
logical response to the material, ${ }^{26,34,35}$ we deduce that an increase in the degree of conversion can reduce the cytotoxicity of the sealers.

In this study the reactions of polymerization resulted not complete following the first irradiation time: the DC progressively increased as a function of the irradiation and its values after each subsequent irradiation contributed to a reduction of the percentage of unreacted monomers. Therefore the results of our experiment are qualitatively in accordance with the study of Mazinis and Lambrianides, according to which there's not correlation between setting time and setting conversion of endodontic sealers as the setting conversion of the material continues after the setting time (that is the time when the material harden). ${ }^{14}$

\section{CONCLUSIONS}

According to the results obtained in this study, it could be concluded that:

- Either using Mini LED or UltraLume LED 5 as light unit, EndoREZ in association with the accelerator exhibits significantly higher values of DC than EndoREZ and RealSeal, both without catalysts;

- The use of the Mini LED produced higher DC than the UltraLume LED 5, both in the EndoREZ with or without Accelerator and in the RealSeal;

- The relative DC progressively increased in all the specimens tested from the first to the fourth irradiation;

- Only further studies can evaluate if the use of EndoREZ with the addition of the accelerator may represent an advantage or a disadvantage for its clinical use.

\section{REFERENCES}

1. Baumgartner JC, Siqueira JR JF, Sedgley CM, Kishen A. Microbiology of endodontic disease. In: INGLE JI, BAKLAND LK, BAUMGARTNER JC. Ingle's Endodontics 6. Hamilton, Ontario, CA: BC Decker, 2008;257.

2. Sly MM, Moore BK, Platt JA, Brown CE. Push-out bond strength of a new endodontic obturation system (Resilon/ Epiphany). J Endod 2007;33:160-162.

3. Dultra F, Barroso JM, Carrasco LD, Capelli A, Guerisoli DM, Pécora JD. Evaluation of apical microleakage of teeth sealed with four different root canal sealers. J Appl Oral Sci $2006 ; 14: 341-345$.
4. Adanir N, Cobankara FK, Belli S. Sealing properties of different resin-based root canal sealers. J Biomed Mater Res B Appl Biomater 2006;77:1-4.

5. De Almeida WA, Leonardo MR, Tanomaru Filho M, Silva LA. Evaluation of apical sealing of three endodontic sealers. Int Endod J 2000;33:25-27.

6. Da Silva Neto UX, De Moraes IG, Westphalen VP, Menezes $R$, Carneiro E, Fariniuk LF. Leakage of 4 resin-based rootcanal sealers used with a single-cone technique. Oral Surg Oral Med Oral Pathol Oral Radiol Endod 2007; 104:e53-e57.

7. Tay FR, Loushine RJ, Monticelli F, Weller N, Breschi L, Ferrari M, Pashley D. Effectiveness of resin coated guttapercha cones and a dual-cured, hydrophilic methacrylate resin-based sealer in obturating root canals. $J$ Endod 2005;31:695-664.

8. Zmener O, Banegas G, Pameijer $\mathrm{CH}$. Bone tissue response to a methacrylate-based endodontic sealer: a histological and histometric study. J Endod 2005;31:457-459.

9. Zmener 0 , Pameijer $\mathrm{CH}$. Clinical and radiographic evaluation of a resin-based root canal sealer. Am J Dent 2004;17:19-22.

10. Cobankara FK, Orucoglu H, Ozkan HB, Yildirim C. Effect of immediate and delayed post preparation on apical microleakage by using methacrylate-based EndoREZ sealer with or without accelerator. J Endod 2008;34:1504-1507.

11. Antonucci JM, Toth EE. Extent of polymerization of dental resins by differential scanning calorimetry. J Dent Res 1983;62:121-125.

12. Atai M, Ahmadi M, Babanzadeh S, Watts DC. Synthesis, characterization, shrinkage and curing kinetics of a new low-shrinkage urethane dimethacrylate monomer for dental applications. Dent Mater 2007;23:1030-1041.

13. Emami N, Söderholm KJ. Influence of light-curing procedures and photo-initiator/co-initiator composition on the degree of conversion of light-curing resins. J Mater Sci Mater Med 2005;16:47-52.

14. Mazinis E, Eliades G, Lambrianides T. An FTIR study of the setting reaction of various endodontic sealers. J Endod 2007;33:616-620.

15. Allan NA, Walton RE, Schaeffer M. Setting times for endodontic sealers under clinical usage and in vitro conditions. J Endod 2001;27:421-423.

16. Teixeira FB, Teixeira ECN, Thompson JY, Trope M. Fracture resistance of roots endodontically treated with a new resin filling material. J Am Dent Assoc 2004;135:646-652.

17. Kurachi C, Tuboy AM, Magalhães DV, Bagnato VS. Hardness evaluation of a dental composite polymerized with experimental LED-based devices. Dent Mater 2001;17:309315 
18. Cadenaro M, Breschi L, Antoniolli F, Mazzoni A, Di Lenarda R. Influence of whitening on the degree of conversion of dental adhesives on dentin. Eur J Oral Sci 2006;114:257262.

19. Cadenaro M, Antoniolli F, Sauro S, Tay FR, Di Lenarda R, Prati C, Biasotto M, Contardo L, Breschi L. Degree of conversion and permeability of dental adhesives. Eur J Oral Sci 2005; 113:525-530.

20. Ma Z, Gao J. Curing kinetics of o-cresol formaldehyde epoxy resin and succinic anhydride system catalyzed by tertiary amine. J Phys Chem B 2006;110:12380-12383.

21. Zmener O, Pameijer $\mathrm{CH}$, Serrano SA, Vidueira M, Macchi $R L$. Significance of moist root canal dentin with the use of methacrylate-based endodontic sealers: an in vitro coronal dye leakage study. J Endod 2008;34:76-79.

22. Perdigão J, Lopes MM, Gomes G. Interfacial adaptation of adhesive materials to root canal dentin. $J$ Endod 2007;33:259-263

23. Hayakawa T, Takahashi K, Kikutake K, Yokota I, Nemoto K. Analysis of polymerization behavior of dental dimethacrylate monomers by differential scanning calorimetry. J Oral Sci 1999;41:9-13.

24. Knezević A, Tarle Z, Meniga A, Sutalo J, Pichler G, Ristić M. Degree of conversion and temperature rise during polymerization of composite resin samples with blue diodes. $J$ Oral Rehabil 2001;28:586-591.

25. Alpoz AR, Ertugrul F, Cogulu D, Ak AT, Tanoglu M, Kaya E. Effects of light curing method and exposure time on mechanical properties of resin based dental materials. Eur $J$ Dent 2008;2:37-42.

26. Ak AT, Alpoz AR, Bayraktar O, Ertugrul F. Monomer release from resin based dental materials cured with LED and halogen lights. Eur J Dent 2010;4:34-40.

27. Pomrink GJ, Dicicco MP, Clineff TD, Erbe EM. Evaluation of the reaction kinetics of CORTOSSTM, a thermoset cortical bone void filler. Biomaterials 2003;24:1023-1031.

28. Schneider LF, Pfeifer CS, Consani S, Prahl SA, Ferracane $J L$. Influence of photoinitiator type on the rate of polymerization, degree of conversion, hardness and yellowing of dental resin composites. Dent Mater 2008;24:1169-1177.

29. Morgan DR, Kalachandra S, Shobha HK, Gunduz N, Stejskal EO. Analysis of a dimethacrylate copolymer (Bis-GMA and TEGDMAl network by DSC and ${ }^{13} \mathrm{C}$ solution and solidstate NMR spectroscopy. Biomaterials 2000;21:1897-1903.

30. Faria-E-Silva AL, Piva E, Moraes RR. Time-dependent effect of refrigeration on viscosity and conversion kinetics of dental adhesive resins. Eur J Dent 2010;4:150-155.

31. Leonard DL, Charlton DG, Roberts HW, Cohen ME. Polymerization efficiency of LED curing lights. J Esthet Restor Dent 2002;14:286-295.
32. Nomura Y, Teshima W, Tanaka N, Yoshida Y, Nahara Y, Okazaki M. Thermal analysis of dental resins cured with blue light-emitting diodes (LEDs). J Biomed Mater Res 2002;63:209-213.

33. Pfeifer CS, Ferracane JL, Sakaguchi RL, Braga RR. Factors affecting photopolymerization stress in dental composites. J Dent Res 2008;87:1043-1047.

34. Brackett MG, Bouillaguet S, Lockwood PE, Rotenberg S, Lewis JB, Messer RL, Wataha JC. In vitro cytotoxicity of dental composites based on new and traditional polymerization chemistries. J Biomed Mater Res B Appl Biomater 2007;81:397-402.

35. Goldberg M. In vitro and in vivo studies on the toxicity of dental resin components: a review. Clin Oral Investig $2008 ; 12: 1-8$ 